FACULDADE DE CIÊNCIAS ECONô MICAS dA UFRGS
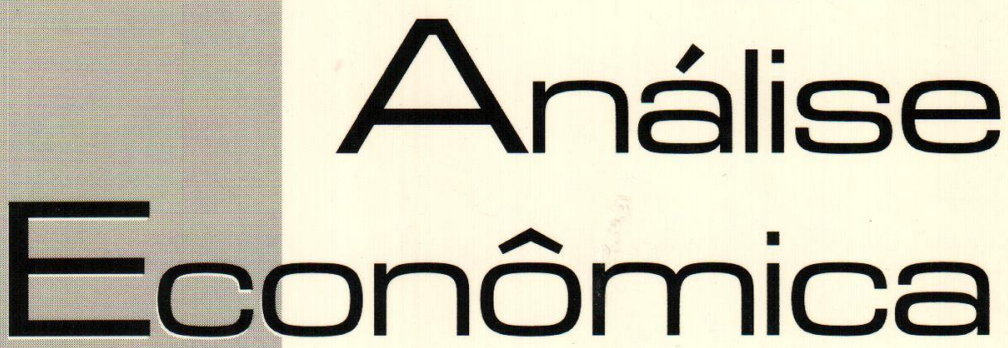

ESCOLHA DE PORTFÓLIO, INVESTIMENTO E NĀO. NEUTRALIDADE DA MOEDA

JOSÉ LUIS OREIRO

REFORMAS NA AROUITETURA FINANCEIRA INTERNACIONAL: NOVIDADES NO FRONT? ANDRÉ MOREIRA CUNHA

MERCOSUR'S CHANGE IN TRADE PATTERNS ANDRÉ FILIPE ZAGO DE AZEVEDO

O IMPACTO DA COMPOSICÃO SETORIAL, DOS FLUXOS INTRA-SETORIAIS E DA ABERTURA COMERCIAL NA PARTICIPAÇÁO DE MERCADO DAS EXPORTAÇÓES BRASILEIRAS

CLÉSIO LOURENÇO XAVIER E EMERSON FERNANDES MARÇAL

O EFEITO BALASSA-SAMUELSON E A PARIDADE DO PODER DE COMPRA NA ECONOMIA BRASILEIRA CLÁUDIO ROBERTO FÓFFANO VASCONCELOS

CICLOS Y FLUCTUACIONES FINANCIERAS: LA IRREGULAR DINÁMICA ECONÓMICA

SARY LEVY-CARCIENTE

O PENSAMENTO DE KARL POPPER: AS DIFERENTES INTERPRETACÓES DOS METODÓLOCOS DA CIENCIA ECONÓMICA

SOLANCE REGINA MARIN E RAMÓN GARCÍA FERNÁNDEZ

A EXPANSÃO DO ESCOPO TEMÁTICO DAS NEGOCIAÇÓES COLETIVAS DE TRABALHO CARLOS HENRIQUE HORN

EFEITOS DO CAPITAL SOCIAL E DO CAPITAL POLITICO NO DESENVOLVIMENTO ECONOMMICO SIMULAÇOES PARA PAISES E ESTADOS BRASILEIROS

RONALDO A. ARRAES, RICARDO CANDÉA S. BARRETO E VLADIMIR KÜHL TELES

O PROBLEMA DE RISCO MORAL NO MERCADO BRASILEIRO DE ASSISTÊNCIA MÉDICA SUPLEMENTAR LUCIANA PINTO DE ANDRADEE SABINO DA SILVA PÓRTO JÚNIOR

ANO

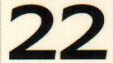

No 41

Março, 2004 
Universidade Federal do Rio Grande do Sul

Reitora: Profo. Wrana Maria Panizzi

Faculdade de Ciencias ECONOMICAS

Diretora: Prof Pedro César Dutra Fonseca

Centro de Estudos e Pesquisas Economicas

Diretor: Prof. Gentil Corazza

Departamento de Ciencias Económicas

Chefe: Prof. Ricardo Dathein

Curso de Pós-Graduação em Economia

Coordenador: Prof. Eduardo Pontual Ribeiro

Programa de Pó-Graduação em Desenvolvimento Rural

Coordenador: Prof. Jalcione Almeida

CONSElHo EDITORIAL:

Carlos G. A. Mielitz Netto (UFRGS), Eduardo A. Maldonado Filho (UFRGS), Eduardo P. Ribeiro (UFRGS), Eleutério F. S. Prado (USP), Eugênio Lagemann (UFRGS), Fernando Cardim de Carvalho (UFRJ), Fernando Ferrari Filho (UFRGS), Fernando de Holanda Barbosa (FGV/RJ), Flávio Vasconcellos Comim (UFRGS), Gentil Corazza (UFRGS), Giácomo Balbinotto Netto (UFRGS), Gustavo Franco (PUC/RJ), Jan A. Kregel (UNCTAD), João Rógério Sanson (UFSC), Joaquim Pinto de Andrade (UnB), Jorge Paulo Araújo (UFRGS), Juan H. Moldau (USP), Marcelo S. Portugal (UFRGS), Maria Alice Lahorgue (UFRGS), Paul Davidson (University of Tennessee), Paulo D. Waquil (UFRGS), Pedro C. D. Fonseca (UFRGS), Philip Arestis (Levy Economics Institut of Bard College), Roberto C. de Moraes (UFRGS), Ronald Otto Hillbrecht (UFRGS), Sabino da Silva Porto Jr. (UFRGS), Stefano Florissi (UFRGS) e Werner Baer (University of Illinois at Urbana-Champaign).

COMISSĀO EDITORIAL:

Eduardo Augusto Maldonado Filho, Fernando Ferrari Filho, Gentil Corazza, Marcelo Savino Portugal, Paulo Dabdab Waquil e Roberto Camps Moraes.

EDITOR: Prof. Fernando Ferrari Filho

Editor Adjunto: Prof. Gentil Corazza

SeCretária: Paulo Roberto Eckert

REVISÃO DE TEXTOS: Vanete Ricacheski

EdITORAÇÃo Eletrónca: Vanessa Hoffmann de Quadros

Fundador: Prof. Antônio Carlos Santos Rosa

Os materiais publicados na revista Análise Econômica são da exclusiva responsabilidade dos autores. É permitida a reprodução total ou parcial dos trabalhos, desde que seja citada a fonte. Aceita-se permuta com revistas congêneres. Aceitam-se, também, livros para divulgação, elaboração de resenhas e recensões. Toda correspondência, material para publicaçāo (vide normas na terceira capa), assinaturas e permutas devem ser dirigidos ao seguinte destinatário:

PROF. FERNANDO FERRARI FILHO Revista Análise Econômica - Av. João Pessoa, 52 CEP 90040-000 PORTO ALEGRE - RS, BRASIL Telefones: (051) 316-3513 - Fax: (051) 316-3990

Análise Econômica E-mail: rae@ufrgs.br

Ano 22, $n^{\circ} 41$, março, 2004 . Porto Alegre

Faculdade de Ciências Econômicas, UFRGS, 2004

Periodicidade semestral, março e setembro.

Tiragem: 500 exemplares

1. Teoria Econômica - Desenvolvimento Regional -

Economia Agrícola - Pesquisa Teórica e Aplicada -

Periódicos. I. Brasil

Faculdade de Ciências Econômicas,

Universidade Federal do Rio Grande do Sul. 


\title{
Escolha de Portfólio, Investimento e Não-Neutralidade da Moeda \\ Uma crítica a tese neoclássica de neutralidade da moeda no longo prazo
}

José Luis Oreiro ${ }^{* *}$

\begin{abstract}
Resumo: o presente artigo tem por objetivo fazer uma avaliação crítica da tese neoclássica de que a moeda é não-neutra apenas no curto prazo. Nesse contexto, iremos argumentar que a neutralidade da moeda no longo prazo é um resultado pouco robusto, pois depende criticamente da hipótese de que a produtividade marginal do capital é constante e independente das decisóes de portfólio dos agentes. Em outras palavras, a neutralidade da moeda no longo prazo não exige apenas que a economia esteja operando com plena utilização da capacidade produtiva - ou pleno emprego da força de trabalho - mas também que a produtividade marginal do capital não seja afetada por variações do investimento planejado. Se essa condição não for atendida, um aumento once-and-for-all da oferta de moeda (um deslocamento da LM para a direita) irá produzir uma redução da taxa real de retorno dos ativos de capital, mesmo no caso em que a economia esteja operando com pleno emprego da força de trabalho. Isso ocorre porque um aumento da oferta de moeda irá produzir uma situação inicial de excesso de oferta de moeda que induzirá os agentes a se livrar dessa moeda em excesso através de um aumento das compras de bens de capital, ou seja, através de um aumento do investimento. Com a economia operando em pleno emprego, o preço de oferta do equipamento de capital recentemente produzido irá aumentar, fazendo com que haja uma redução da taxa real de retorno dos ativos em consideração. A moeda é não-neutra no longo prazo porque ela é capaz de influenciar a composição - embora não o nível absoluto - do produto real, bem como a taxa real de retorno do investimento em capital fixo.
\end{abstract}

Palavras-Chave : Escolha de portfólio, eficiência marginal do capital, política monetária.

\footnotetext{
" Artigo baseado no capítulo 1 da tese de doutorado do autor intitulada "Incerteza, Instabilidade Macroeconômica e Crescimento Endógeno: ensaios em teoria pós-keynesiana" feita sob orientação do Prof. Dr. Fernando Cardim de Carvalho.

" Doutor em Economia (IE/UFRJ) e Professor Adjunto do Departamento de Economia da Universidade Federal do Paraná.E-mail: joreiro@sociais.ufpr.br e jlcoreiro@aol.com. O autor agradece ao suporte financeiro do $\mathrm{CNPQ}$.
} 
Abstract: The objective of the present article is to do a critical evaluation of the neoclassical thesis of long-run neutrality of money. We will argue that long-run neutrality of money is not a general result since it depends critically on a very particular hypothesis, that is, marginal productivity of capital is exogenous and independent of portfolio decisions taken by economic agents. In other words, long-run neutrality of money is not a direct result of the supposed convergence of real income towards its full employment level in the long-run; but also depends on the assumption of exogenous marginal productivity of capital. If this condition does not hold, than a once-and-for-all increase in money supply (a leftward shift of LM curve) will produce a reduction in real rate of return of capital assets, even if real income is on its full-employment level. This happens because an increase in money supply will produce a disequilibrium in money market, more precisely, a situation of excess supply of money which will induce economic agents to get ride of this excessive money by means of increasing their purchases of capital goods. Since real income is on its full-employment level, an increase in demand for capital goods will result in an increase of capital goods prices, thereby reducing real rate of return of capital assets. Money is not neutral in the long-run since it is capable to influence the composition of real output, even if its absolute level is constant, and the real rate of return of capital assets.

Key-Words: Porfolio Choice, Marginal Efficiency of Capital, Monetary Policy.

JEL Classification: E12; E44; E52.

\section{Introdução}

Um dos principais argumentos que Keynes apresentou como crítica a assim chamada Teoria Clássica era de que a mesma seria incapaz de explicar a racionalidade da demanda de moeda como reserva de valor. Nas suas palavras:

Money, it is well know, serves two principal purposes. By acting as a money of account it facilitates exchanges without its being necessarily that it should ever itself come into the picture as a substantive object [...] In the second place, it is a store of wealth. So we are told, without a smile on the face. But in the world of the classical economy what an insane use to which to put it! For it is a recognized characteristic of money as a store of wealth that it is barren; whereas practically every other form of storing wealth yields some interest or profit. Why should anyone outside a lunatic asylum wish to use money as a store of wealth? (CWJMK, Vol. XIV, p.115-116) 
Essa incapacidade de explicar a racionalidade da demanda de moeda como reserva de valor resultaria, por sua vez, da desconsideração da incerteza implícita nos processos de tomada de decisão (Ibid, p.113-115). O "erro" da Teoria Clássica estaria precisamente em ignorar o fato de que o desejo de manter moeda em portfólio resulta da desconfiança que os agentes têm em suas próprias previsões sobre o futuro. Segundo Keynes:

... our desire to hold money as a store of wealth is a barometer of the degree of our distrust of our own calculations and conventions concerning the future. Even though this feeling about money is itself conventional or instinctive, it operates [...] at a deeper level of our motivation [...] The possession of actual money lulls our disquietude; and the premium which we require to make us to part with money is the measure of the degree of our disquietude (Ibid, p. 116).

No mundo suposto pela Teoria Clássica, a moeda não desempenharia um papel essencial no sistema econômico, uma vez que ela serviria unicamente como meio de troca (Ibid, p.115). Nesse contexto, ela não teria nenhuma influência sobre as "motivações" e as decisões dos agentes econômicos; sendo, portanto, neutra. A moeda seria apenas um "véu" que encobre o lado real da economia, o qual é definido de forma independente de aspectos monetários. Essa posição de Keynes encontra suporte nos autores da própria escola clássica, como, por exemplo, Pigou, o qual afirma que:

Monetary facts [...] differ from 'real' facts and happenings in that unlike these they have no direct significance for economic welfare. Take the real facts and happenings away, and the monetary facts and happenings necessarily vanish with; but take money away and, whatever else might follow, economic life would not become meaningless: there is nothing absurd about the conception of a self-sufficing family, or village group, without any money at all. In this sense money is clearly a veil. It does not comprise any of essentials of economic life (apud Lima, 1992, p.111).

Entretanto, o suposto de "neutralidade da moeda" pela Teoria Clássica era, segundo Keynes, a razão pela qual a mesma não seria capaz de explicar a ocorrência de crises e de desemprego crônico da força de trabalho. A explicação desses fenômenos exigiria, portanto, uma teoria que fosse capaz de dar a moeda um papel relevante no sistema econômico, o que só seria possível mediante a consideração explícita da incerteza envolvida no processo de tomada de 
decisões. Em outras palavras, era necessário uma teoria na qual a moeda fosse não-neutra. Nas palavras de Keynes:

In my opinion the main reason why the problem of crisis is unsolved, or at any rate why this theory is so unsatisfactory, is to be found in the lack of might be termed a monetary theory of production [...] the theory which I desiderate would deal with an economy in which money plays a part of its own and affects motives and decisions and is, in short, one of the operative factors in the situation, so that the course of the events cannot be predicted, either in the long period or in the short, without a knowledge of the behavior of money between the first state and the last (CWJMK, Vol. XIII, p.408-409).

Poucos autores negariam a validade das críticas de Keynes à Teoria Clássica, particularmente no que se refere ao tratamento conferido pela mesma à moeda e à incerteza que circunda o processo de tomada de decisões ${ }^{1}$. Contudo, esse mesmo consenso seria mais difícil de ser obtido com relação à versão mais sofisticada dessa teoria que surgiu e se desenvolveu nas décadas posteriores à publicação da Teoria Geral do Emprego, do Juro e da Moeda - doravante TG - ou seja, com relação a assim chamada Síntese Neoclássica.

Primeiramente se poderia argumentar que a incerteza foi explicitamente incorporada ao processo de tomada de decisão desde os trabalhos seminais de Von Neumann-Morgenstern (1944) e Savage (1954). Nesse contexto, a decisão sob incerteza tem sido tratada como uma situação na qual o agente econômico associa uma distribuição de probabilidades (objetiva ou subjetiva) aos resultados possíveis dos diversos cursos de ação. Sob certas condições pode-se demonstrar que (i) as preferências dos agentes a respeito dos diversos cursos de ação podem ser representadas por intermédio de uma função utilidade esperada; e (ii) o indivíduo escolhe aquele curso de ação que lhe proporciona a maior utilidade esperada possível.

Por outro lado, o trabalho seminal de Tobin (1958) poderia ser citado como um exemplo de como a teoria neoclássica é capaz de explicar a função da moeda como reserva de valor. De fato, no modelo de Tobin, os tomadores de decisão demandam moeda como

\footnotetext{
${ }^{1}$ Milgate (1982) é uma notável exceção a essa linha de argumentação. Segundo esse autor, a teoria clássica, ao menos nos trabalhos de Hawtrey e Robertson, teria explicitamente levado em conta a presença de incerteza no processo de tomada de decisão (Ibid, $\mathrm{p}_{\mathrm{i}} 150$ ). Isso porque tais autores teriam reconhecido formalmente a dependência de tais decisōes com relação às expectativas sobre os valores futuros das variáveis econômicas.
} 
uma forma de se proteger contra o risco de perda de capital em função de variações imprevistas e desfavoráveis dos preços dos demais ativos. A demanda de moeda como reserva de valor resulta, nesse contexto, da "aversão ao risco" por parte dos agentes econômicos.

Essa mesma aversão ao risco faz com que a moeda e os demais ativos sejam substitutos imperfeitos entre si no portfólio dos agentes. Essa hipótese combinada com o fato de que a taxa nominal de retorno da moeda é igual a zero daria à política monetária a capacidade de (i) alterar as taxas reais de retorno dos diversos ativos no curto prazo e (ii) modificar as ofertas relativas dos diversos ativos no longo prazo. Nesse caso, a teoria neoclássica seria capaz de demonstrar que (a) um aumento once-and-for-all da oferta de moeda pode induzir um aumento do nível de renda e de emprego no curto prazo à medida que induz os agentes econômicos a aumentar o investimento em bens de capital; e (b) um aumento da taxa de crescimento da oferta de moeda pode produzir um aumento do nível de renda per capita e de capital per capita de steady-state, uma vez que induz os tomadores de decisão a substituir moeda por capital em seus portfólios, processo esse que, sob certas condições, produz um aumento da taxa de poupança. Em outros termos, a teoria neoclássica admite a não-neutralidade da moeda no curto-prazo (mas não no longo prazo) e a super não-neutralidade da moeda no longo prazo.

Isso posto, o presente artigo tem por objetivo fazer uma avaliação crítica da tese neoclássica de que a moeda é não-neutra apenas no curto prazo. Nesse contexto, iremos argumentar que a neutralidade da moeda no longo prazo é um resultado pouco robusto, pois depende criticamente da hipótese de que a produtividade marginal do capital é constante e independente das decisões de portfólio dos agentes. Em outras palavras, a neutralidade da moeda no longo prazo não exige apenas que a economia esteja operando com plena utilização da capacidade produtiva - ou pleno emprego da força de trabalho - mas também que a produtividade marginal do capital não seja afetada por variações do investimento planejado. Se essa condição não for atendida, uma variação once-and-for-all da oferta de moeda (um deslocamento da LM para a direita) irá produzir uma redução da taxa real de retorno dos ativos de capital, mesmo no caso em que a economia esteja operando com pleno emprego da força de trabalho. Daqui se segue, nessas condições, a moeda é não-neutra no longo prazo. 
Isso ocorre porque uma expansão monetária irá produzir uma situação inicial de excesso de oferta de moeda que induzirá os agentes a se livrar dessa moeda em excesso através de um aumento das compras de bens de capital, ou seja, através de um aumento do investimento. Com a economia operando em pleno emprego, o preço de oferta do equipamento de capital recentemente produzido irá aumentar, fazendo com que haja uma redução da taxa real de retorno (eficiência marginal do capital) dos ativos em consideração. A moeda é não-neutra no longo prazo porque ela é capaz de influenciar a composição - embora não o nível absoluto - do produto real (ou seja, a divisão do produto entre produção de bens de consumo e produção de bens de capital), bem como a taxa real de retorno do investimento em capital fixo.

O artigo está estruturado em quatro seções, incluindo a introduçāo. A segunda seção apresenta o modelo moeda-títulos-capital desenvolvido por Tobin (1969), o qual é o fundamento teórico para a tese de neutralidade da moeda no longo prazo. A terceira apresenta uma reformulação desse modelo com base em Petenatti (1977), no qual se demonstra que o resultado Tobiniano de neutralidade de longo prazo da política monetária não se segue caso a "eficiência marginal do capital" seja influenciada por variações no investimento planejado pelas firmas. A quarta seção resume as conclusões obtidas ao longo do artigo.

\section{Escolha de Portfólio e Não-Neutralidade da Moeda: o modelo moeda-títulos-capital de Tobin}

Consideremos uma economia com três ativos, a saber: moeda, títulos do governo e capital, sendo que esses ativos são substitutos próximos entre si. A moeda também é objeto de decisão de portfólio uma vez que ela é o único ativo que está totalmente protegido contra o risco de perda de capital. Nesse contexto, se os indivíduos possuírem algum grau de aversão ao risco, então eles irão manter uma parte de sua riqueza sob a forma de moeda como uma estratégia defensiva contra o risco em consideração.

A hipótese de que os referidos ativos são substitutos próximos entre si garante que a demanda por qualquer um dos mesmos será influenciada não só pela taxa de retorno do próprio ativo, como 
também pela taxa de retorno de todos os demais ativos. Em particular, se valer a hipótese de substituição bruta, segue-se que as derivadas parciais das funçóes de demanda de cada um dos ativos em consideração com respeito a taxa de retorno dos demais ativos será não-positiva; em outras palavras, se a taxa de retorno do ativo $j$ aumentar, entāo a demanda do ativo $i$ irá diminuir ou permanecer constante.

Com base nessas informações, podemos apresentar as funções de demanda dos ativos em consideração por intermédio do seguinte conjunto de equaçōes:

$$
\begin{aligned}
& f_{l}\left(r_{k}, r_{m}, r_{s}, Y / W\right) W=(q K)^{d} \\
& f_{2}\left(r_{k}, r_{m}, r_{s^{\prime}} Y / W\right) W=(M / P)^{d} \\
& f_{3}\left(r_{k}, r_{m}, r_{s^{\prime}}, Y / W\right) W=(S / P)^{d} \\
& r_{k}=R / q \\
& r_{m}=r_{m}^{-} \rho^{*} \\
& r_{s}=r_{s}^{-}-\rho^{*}
\end{aligned}
$$

onde: W é o valor real do estoque de riqueza possuído pelos indivíduos, $M$ é a quantidade nominal de moeda, $S$ é o valor nominal das obrigaçōes, $K$ é o valor real do estoque de capital, $r_{k}$ é a taxa real de retorno sobre o capital, $r_{m}$ é a taxa real de retorno sobre a moeda, $r_{s}$ é a taxa real de retorno sobre as obrigaçōes, $r_{m}^{\prime}$ é a taxa nominal de retorno sobre a moeda, $r_{s}^{\prime}$ é a taxa nominal de retorno sobre as obrigaçōes, $\rho^{*}$ é a taxa esperada de inflação, $\mathrm{R}$ é a produtividade marginal do capital e $q$ é a relação entre o valor de mercado das ações de uma empresa e o custo de reposição do seu capital.

No sistema de equações (1)-(6) iremos considerar que a renda é um dado exógeno, ou seja, iremos desconsiderar qualquer influência que a decisão de composição de portfólio tenha sobre o nível de renda agregada. Essa hipótese se fundamenta no assim chamado teorema da separação, o qual afirma que decisões de dispêndio e de composição de portfólio podem ser analisadas separadamente entre $\mathrm{si}^{2}$. Além disso, iremos considerar que as proporções dos diver-

\footnotetext{
${ }^{2}$ Nas palavras de Tobin: "The Key behavioral assumption of this procedure is that spending decisions and portfolio decisions are independent - specifically that the decisions about the accumulation of wealth are separable from the decisions about its allocation. As savers, people decide how much to add to their wealth, as portfolio managers, they decide how to distribute among available assets and debts the net worth they already have" (Tobin, 1969, p. 323).
} 
sos ativos que os indivíduos desejam manter em seus portfólios não são influenciadas por variações no estoque de riqueza ${ }^{3}$.

O equilíbrio nos mercados de cada um dos ativos em consideração requer que as quantidades demandadas de cada um desses ativos sejam iguais aos estoques disponíveis dos mesmos. Além disso, o valor dos estoques existentes dos ativos em consideração deve ser igual ao valor do estoque de riqueza possuído pelos indivíduos. Temos, assim, o seguinte sistema de equações:

$$
\begin{aligned}
& f_{t}\left(r_{k}, r_{m^{\prime}}, r_{s}, Y / W\right) W=q K \\
& f_{2}\left(r_{k}, r_{m}, r_{s}, Y / W\right) W=M / P \\
& f_{3}\left(r_{k}, r_{m}, r_{s}, Y / W\right) W=S / P \\
& r_{k}=R / q \\
& r_{m}=r_{m}-\rho^{*} \\
& r_{s}=r_{s}^{-}-\rho^{*} \\
& W=q K+(M+S) / P
\end{aligned}
$$

Com base na equação (13), podemos observar que o sistema formado pelas equações (7)-(13) só possui 6 equaçōes linearmente independentes. De fato, a equação (13) garante que se os mercados dos ativos capital e obrigações estiverem em equilíbrio, então o mercado do ativo moeda também o estará.

As variáveis exógenas do referido sistema de equaçōes são: $\mathrm{Y}, \mathrm{K}$, $\mathrm{M}, \mathrm{S}, \mathrm{R}, \rho^{*}, \mathrm{P}$ e $\mathrm{r}_{\mathrm{m}}^{\prime}$; as variáveis endógenas são: $\mathrm{r}_{\mathrm{k}}, \mathrm{r}_{\mathrm{s}}, \mathrm{r}_{\mathrm{m}}, \mathrm{r}^{\prime} \mathrm{s}, \mathrm{q}$ e W. No total temos seis incógnitas a serem determinadas em seis equações linearmente independentes. O sistema tem, em princípio, solução.

A inspeção do sistema em consideração mostra que o mesmo é bloco-recursivo, ou seja, a interdependência entre as equações que o constituem não é geral (cf. Sargent, 1987, p.23). As equaçōes (7), (8), (9), (10) e (13) são suficientes para determinar $W, r_{m}, r_{k}$ e $r_{s}$; dado $r_{s}$ determina-se $r^{\prime}{ }_{s}$ em (12) e dado $r_{k}$ determina-se $q$ em (10).

A partir das equaçōes (8) e (9), tomando como dado $r_{m}$ a partir da equação (11), podemos determinar os locus geométricos das combinações entre $q$ e $r_{s}$ para as quais os mercados de moeda - locus

\footnotetext{
${ }^{3}$ Essa suposição só é válida num contexto em que o grau de aversão absoluta ao risco é independente de variações no estoque de riqueza. Para que isso ocorra, contudo, a utilidade esperada não pode ser uma funçāo quadrática.
} 
MM - e de obrigações - locus SS - estão em equilíbrio. De fato, diferenciando (8) e (9) com respeito a $q$ e $r_{s}$ temos:

$$
\begin{array}{lll}
\frac{\partial q}{\partial r_{s}}=\frac{\left(\frac{\partial f_{2}}{\partial r_{s}}\right)}{\left(\frac{R}{q^{2}}\right)\left(\frac{\partial f_{3}}{\partial r_{k}}\right)}>0 & (14 a) & \text { [Locus M } \left.M^{\prime}\right] \\
\frac{\partial q}{\partial r_{s}}=\frac{\left(\frac{\partial f_{3}}{\partial r_{s}}\right)}{\left(\frac{R}{q^{2}}\right)\left(\frac{\partial f_{3}}{\partial r_{k}}\right)}<0 & (14 b) & {\left[\text { Locus } S S^{\prime}\right]}
\end{array}
$$

A determinação de $r_{s}$ e de $q$ pode ser visualizada por intermédio da Figura 1.

\section{Figura 1}

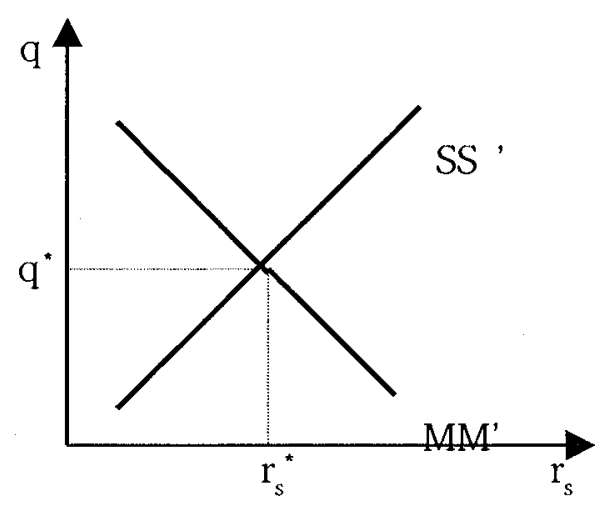

No modelo em consideração, a política monetária é capaz de influenciar a taxa real de retorno sobre os ativos capital e obrigações; em outras palavras, o valor da variável $q$ pode ser influenciado pelas operações de open-market conduzidas pelas autoridades monetárias. Para comprovar esse efeito, consideremos uma situação na qual o Banco Central realiza uma operação de compra das obrigações do governo possuídas pelos indivíduos. Como é bemsabido, as operaçōes de open-market não alteram o estoque de riqueza possuído pelos agentes, ou seja, $\mathrm{DM}=-\Delta \mathrm{S}$. Dessa forma, diferenciando (8) e (19) com respeito a $M, S, r_{s} \cdot e r_{k}$ temos:

$$
f_{2}^{1} d r_{k}+f_{2}^{3} d r_{s}=(1 / P) d M
$$


Colocando $\mathrm{dr}_{\mathrm{s}}$ em evidência na equação (16) e substituindo a equação resultante em (15), temos após todos os algebrismos necessários que:

$$
\frac{\partial r_{k}}{\partial M}=\frac{\left(\frac{1}{P}\right)\left(1+\left(\frac{f_{2}^{3}}{f_{3}^{3}}\right) f_{3}^{1}\right)}{\left(f_{2}^{1}-\frac{f_{2}^{3}}{f_{3}^{3}}\right)}
$$

onde: $\mathrm{f}_{\mathrm{i}}^{\mathrm{j}}<0$ se $\mathrm{i} \neq \mathrm{j}$, e $\mathrm{f}_{\mathrm{i}}{ }^{\mathrm{j}}>0$ se $\mathrm{i}=\mathrm{j}$.

$\mathrm{Na}$ equação (17) observa-se que o numerador é claramente positivo, ao passo que o denominador tem sinal ambíguo. Para que um aumento da oferta de moeda reduza a taxa real de retorno sobre o capital é, portanto, necessário que o denominador seja negativo. Isso irá ocorrer se $f_{2}{ }^{1}>\left(f_{2}{ }^{3} / f_{3}^{3}\right)$, ou seja, se moeda e capital forem fortemente substituíveis entre si. Supondo que essa condição seja atendida, temos que um aumento da oferta de moeda através das operações de open-market irá reduzir a taxa real de retorno sobre o capital, ou seja, irá aumentar o valor da variável $q$. Isso ocorre porque a operação de open-market reduziu o estoque de obrigaçōes relativamente ao estoque dos demais ativos existentes na economia. Essa redução produziu um aumento do preço das obrigações, ou seja, uma redução de sua taxa de retorno. Dada a hipótese de substitubilidade bruta entre os diversos ativos, uma redução da taxa de retorno sobre as obrigações estimula positivamente a demanda pelo ativo capital, o que deverá elevar o preço de mercado dos direitos sobre a posse do mesmo, isto é, o preço das açōes.

O aumento do valor da variável $q$ irá sinalizar para os empresários que eles podem aumentar lucrativamente o seu estoque de capital, ou seja, irá estimular o dispêndio de investimento em capital fixo ${ }^{5}$. Segue-se, portanto, que a política monetária é não-neutra sobre as variáveis reais da economia.

\footnotetext{
${ }^{4}$ Os sobreescritos em cima das variáveis $\mathrm{f}_{\mathrm{i}}, \mathrm{i}=2,3$, representam a derivada parcial da função em consideração com relação à variável $r_{k}$ ( sobreescrito 1 ) e com relação à variável $r_{s}$ (sobreescrito 3).

${ }^{5}$ Estamos considerando que a função investimento é dada pela seguinte equação: $\mathrm{I}=\mathrm{I}(\mathrm{q}-1)$, I $(0)=0, I^{-}>0$. A derivação formal dessa função investimento a pārtir da maximização intertemporal de lucros da firma pode ser obtida em Romer (1996, p.348-351) e Takayama (1993, p. 525-535).
} 
Que ou quais fatores são responsáveis pela não-neutralidade da moeda? O subsistema de equações apresentado em (8) - (9) só permite a determinação de duas taxas reais de retorno; isso porque uma das equações desse sistema é linearmente dependente das outras duas. Sendo assim, é impossivel a determinação endógena e simultânea das taxas de retorno dos ativos moeda, capital e obrigações. Uma dessas taxas de retorno deve ser determinada exogenamente ao modelo. Se a taxa real de retorno sobre a moeda for a variável exógena - isso porque a taxa nominal de retorno sobre esse ativo é fixada institucionalmente em zero - então a taxa de retorno sobre o capital pode ser determinada endogenamente. Nesse caso, as autoridades monetárias, através das operações de open-market podem forçar a divergência entre a taxa real de retorno sobre o capital e a sua produtividade marginal. Ao se criar essa divergência, os empresários se sentirão estimulados a investir mais. Nas palavras de Tobin:

... why is substitution of money for securities via open market purchases expansionary?

It is not because asset No.1 has been called 'money' and asset No.2 "securities". It is not because asset No.1 is a means of payment or has any other intrinsic property asset No.2 lacks (...) These properties has nowhere enter in the analysis, except in the general sense that they explain why assets are no perfect substitutes for each other. The essencial characteristic - the only distinction of money from securities that matters for the results given above - is that the interest rate on money is exogenously fixed by law or convention, while the rate of return on securities is endogenous, market determined" (1971, p.334).

Contudo, se a taxa nominal de retorno sobre a moeda pudesse variar livremente, então a taxa real de retorno sobre o capital poderia ser determinada exogenamente ao modelo. Nesse caso, a política monetária seria incapaz de influenciar a taxa de retorno sobre o capital, ou seja, a moeda seria neutra sobre a decisão de acumulação de capital. Segue-se, portanto, que no modelo de Tobin a não-neutralidade da moeda resulta tão-somente da rigidez institucional da taxa nominal de retorno sobre a moeda, não tendo nenhuma relação direta com a maior "liquidez" desse ativo com relação aos demais ativos.

Tudo o que foi dito até o presente momento se referia ao curto prazo marshalliano. No curto prazo, o estoque de capital existente 
na economia é fixo, podendo ocorrer divergências entre o estoque de capital que os indivíduos desejam reter e o estoque que eles efetivamente possuem. No longo prazo, tais divergências não podem ocorrer, ou seja, $q=1$. Sendo assim, temos o seguinte sistema de equações:

$$
\begin{aligned}
& f_{1}\left(r_{k}, r_{m}, r_{s}, Y / W\right) W=K \\
& f_{2}\left(r_{k}, r_{m}, r_{s}, Y / W\right) W=M / P \\
& f_{3}\left(r_{k}, r_{m}, r_{s}, Y / W\right) W=S / P \\
& r_{k}=R \\
& r_{m}=r_{m}^{\prime}-\rho^{*} \\
& r_{s}=r_{s}^{\prime}-\rho^{*} \\
& W=K^{*}+(M+S) / P
\end{aligned}
$$

As variáveis exógenas desse modelo são: $\mathrm{Y}, \mathrm{K}, \mathrm{M}, \mathrm{S}, \rho^{*}, \mathrm{R}, \mathrm{r}_{\mathrm{m}}{ }_{\mathrm{m}}$; ao passo que as variáveis endógenas são: $r_{k}, r_{m}, r_{s}, r_{s}$, W e P. Como $r_{k}$ e $r_{m}$ estão determinadas pelas equações (21) e (22), respectivamente, segue-se que a taxa real de retorno das obrigações $r_{s}$ pode ser determinada exclusivamente a partir da equação (18). Sendo assim, o valor real da oferta de moeda e da oferta de obrigações estarāo automaticamente determinados a partir das equações. (19) e (20). Nesse contexto, um aumento da oferta de moeda por intermédio de uma operação de open-market não deverá ter nenhum efeito sobre as taxas reais de retorno dos ativos em consideração, de forma que a demanda pelos mesmos não deverá se alterar. Isso posto, o único efeito de um aumento once-and-for-all da oferta de moeda será um aumento exatamente proporcional no nível geral de preços; mantendo assim a oferta real de moeda constante. Segue-se, portanto, que a moeda é neutra no longo prazo.

Entretanto, no modelo em consideração, existe a possibilidade de que a moeda não seja superneutra. Para demonstrar esse ponto, devemos ter inicialmente em conta que, no longo prazo, a seguinte relação deve ser válida:

$$
\rho^{*}=\rho=\Theta
$$

onde: $\rho^{*}$ é a taxa esperada de inflação, $\rho$ é a taxa de inflação efetiva, $\Theta$ é a taxa de cresçimento da oferta de moeda.

Substituindo (25) em (22), conclui-se que um aumento da taxa de crescimento da oferta de moeda deverá produzir uma redução da taxa real de retorno sobre a moeda. Isso, por sua vez, tem impacto sobre a estrutura de demanda pelos diversos ativos existentes na 
economia e sobre a taxa real de retorno das obrigações. De fato, diferenciando (1) com respeito a $r_{m}$ e a $r_{s}$ temos que:

$$
\frac{\partial r_{s}}{\partial r_{m}}=-\frac{\left(\frac{\partial f_{1}}{\partial r_{m}}\right)}{\left(\frac{\partial f_{1}}{\partial r_{s}}\right)}<0
$$

Em palavras: uma redução na taxa real de retorno da moeda deverá produzir um aumento da taxa real de retorno das obrigações.

Definindo-se $m=M / P$ e diferenciando (19) com respeito a $m$ e $\mathrm{r}_{\mathrm{m}}$, obtemos a seguinte expressão:

$$
\frac{\partial m}{\partial r_{m}}=\left\{\frac{\partial f_{2}}{\partial r_{s}} \frac{\partial r_{s}}{\partial m}+\frac{\partial f_{2}}{\partial r_{m}}\right\}>0
$$

A expressão (27) mostra que uma redução da taxa real de retorno sobre a moeda - produzida por um aumento da taxa de crescimento da oferta de moeda - deverá produzir uma redução da oferta real de moeda. Segue-se, portanto, que a política monetária pode, no longo prazo, alterar as ofertas relativas dos diversos ativos (cf. Tobin, 1971, p.335).

\section{Uma crítica ao modelo moeda-obrigações-capital}

O modelo moeda-obrigaçōes-capital desenvolvido na seção anterior tinha como uma de suas características fundamentais o fato de que variações do tipo once-and-for-all do estoque nominal de moeda não tinham nenhum efeito sobre as taxas reais de retorno dos ativos em consideração. Entretanto, esse resultado depende criticamente da hipótese de que a taxa real de retorno sobre o capital é constante $e$ independente do nível de investimento desejado pelos tomadores de decisão. Isso só é verdade, contudo, no caso em que o estoque de capital está crescendo à taxa natural, ou seja, quando a taxa de crescimento do mesmo é igual à soma entre a taxa de crescimento da população e a taxa de crescimento da produtividade do trabalho (cf. Pettenati, 1977, p.358-59). Se essa condição não for atendida, então não será possível tomar a taxa real de retorno do capital como uma constante. 
Em equilíbrio de longo prazo, a taxa real de retorno do capital é igual à eficiência marginal do capital (Ibid, p.358), de forma que os tomadores de decisão ficam indiferentes entre adquirir um bem de capital já existente ou encomendar o mesmo junto aos fabricantes do equipamento de capital. A eficiência marginal do capital é definida como sendo igual à taxa de desconto que equaliza o valor presente dos rendimentos, que se pode obter de um determinado equipamento de capital ao longo de toda a sua vida útil, com o preço de oferta do referido equipamento (cf. Keynes, 1936, p.135). Segundo Keynes, a eficiência marginal do capital é uma função decrescente do nível de investimento, isso porque (i) o preço de oferta é uma função crescente do nível de investimento, devido à existência de rendimentos marginais decrescentes no setor produtor de bens de capital; e (ii) os rendimentos futuros do referido equipamento são uma função decrescente do estoque de capital, uma vez que um aumento do mesmo irá reduzir a "escassez do capital" com relação ao "tamanho do mercado" (cf. Keynes, 1936, p.136) ${ }^{6}$. Sendo assim, podemos apresentar a determinação da eficiência marginal do capital por intermédio da seguinte equação ${ }^{7}$ :

$$
R=R(\phi, I, p) \quad \partial R / \partial \phi>0, \partial R / \partial I<0, \partial R / \partial p<0
$$

Onde: $\phi$ é o estado de expectativas de longo período, I é o volume de investimento desejado pelas firmas, p é o preço de oferta do equipamento de capital (igual ao nível geral de preços, sob a hipótese de que a economia produz um único bem).

\footnotetext{
- Diversos autores têm criticado a idéia de que a eficiência marginal do capital é uma função decrescente do nível de investimento. Robinson, por exemplo, afirma que essa idéia pressupōe que os empresários, no seus cálculos ex-ante a respeito da rentabilidade esperada dos projetos de investimento, estariam levando em conta o acréscimo no custo de produção decorrente da implementação (ex-post) dos referidos projetos (apud Possas, 1986, p.302). Como o aumento do preço de oferta é um resultado ex-post, ele não poderia ser presumido no cálculo ex-ante dos tomadores de decisão (Ibid, p.302). Entretanto, tal como ressalta Possas (1986, 1987), a existência de rendimentos decrescentes no setor produtor de bens de capital não é condição necessária para que a eficiência marginal do capital seja uma função decrescente do nivel de investimento. De fato, se as firmas se defrontam com uma curva de demanda negativamente inclinada pelos seus produtos; entẫo elas antecipam que qualquer acréscimo na capacidade de produção - se efetivamente utilizada - deverá levar a uma redução nos preços e nas margens unitárias de lucro e, portanto, na rentabilidade por unidade de capital. investido. Tal condição é suficiente para produzir uma relaçāo inversa entre a eficiência marginal do capital e o volume de investimento desejado pelas firmas.

${ }^{7}$ Essa equação é tirada de Pettenati (1977).
} 
Para demonstrar que a não-neutralidade da moeda no longo prazo, no caso em que a taxa de retorno do capital é determinada pela equação (53), consideremos uma versão mais simples do modelo moeda-obrigaçôes-capital; mais especificamente, consideremos uma economia que possua apenas dois ativos (moeda e capital) e seja descrita pelo seguinte sistema de equações:

$$
\begin{aligned}
& W=q K+\frac{M}{P} \\
& f_{1}\left(r_{k}, r_{m}, \frac{Y}{W}\right) W=q K \\
& f_{2}\left(r_{k}, r_{m}, \frac{Y}{W}\right) W=\frac{M}{P} \\
& q=\frac{v}{p} \\
& v=\sum_{i=1}^{n} \frac{Z_{i}}{\left(1+r_{k}\right)^{i}} \\
& p=\sum_{i=1}^{n} \frac{Z_{i}}{(1+R)^{i}} \\
& R=r_{k} \\
& R=R(\phi, I, p) \\
& I=(1-c) Y \\
& Y=Y_{f}
\end{aligned}
$$

Onde: $V$ é o preço de demanda dos bens de capital, $p$ é o preço de oferta, $Z_{i}$ é o rendimento esperado do equipamento de capital no período $i$, c é a propensão marginal a consumir, $\mathrm{Y}_{\mathrm{f}}$ é o nível de renda de pleno emprego.

Devido à restrição imposta pela equação (29), segue-se que podemos descartar a equação (30), uma vez que $f_{1}=1-f_{2}$. Como $r_{k}=$ $R$, segue-se também que $\mathrm{q}=1$. Após as substituições necessárias, chegamos ao seguinte sistema de equações:

$$
\begin{aligned}
& f_{2}\left[R, r_{n t}, \frac{Y}{K+\left(\frac{M}{P}\right)}\right]\left(K+\left(\frac{M}{P}\right)\right)=\left(\frac{M}{P}\right) \\
& R=R\left(\phi, P,(1-b) Y_{f}\right)
\end{aligned}
$$


A equação (39a) apresenta as combinações entre a taxa de retorno do capital $\left(\mathrm{r}_{k}=\mathrm{R}\right)$ e o nível geral de preços para as quais o mercado monetário está em equilíbrio. Trata-se de uma versão da curva LM para o modelo em consideração. A equação (39b), por seu turno, apresenta as combinações entre a eficiência marginal do capital e o nível geral de preços para as quais o mercado de bens está em equilíbrio (demanda agregada igual ao nível de renda de pleno emprego). Trata-se de uma versão análoga à curva IS. A determinação do nível de preços e da taxa de retorno do capital para os quais todos os mercados estão simultaneamente em equilíbrio pode ser visualizado por intermédio da Figura 2.

\section{Figura 2}

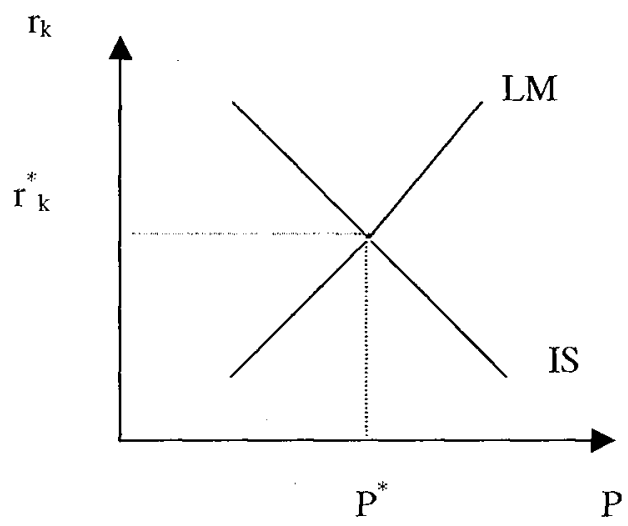

Nesse contexto, consideremos que o governo financia um pacote de obras públicas com emissão monetária, de forma que ocorre um acréscimo do tipo once-and-for-all da oferta de moeda. $\mathrm{O}$ aumento do estoque de moeda irá produzir um desequilíbrio inicial no mercado monetário, uma vez que, aos níveis iniciais de preços e taxa de retorno do capital, a oferta de moeda será maior do que a demanda. O público tentará se livrar da moeda que tem em excesso, comprando bens de capital. O aumento da demanda por bens de capital - num contexto em que a economia opera com plena utilização da capacidade produtiva - irá produzir um aumento do nível geral de preços e, conseqüentemente, do preço de oferta do equipamento de capital recentemente produzido. $\mathrm{O}$ aumento do preço de oferta, por sua vez, irá levar a uma redução da eficiência marginal do capital e, devido às operações de arbitragem, a uma 
redução da taxa real de retorno do capital. Do ponto de vista da composição da produção, irá ocorrer uma redução da produção de bens de consumo e um aumento da produção de bens de capital, ou seja, a expansão monetária gera uma mudança na composição do produto real em direção à produção de máquinas e equipamentos. Segue-se, portanto, que a moeda é não-neutra no longo prazo. A visualização desse processo pode ser feita por intermédio da Figura 3.

\section{Figura 3}

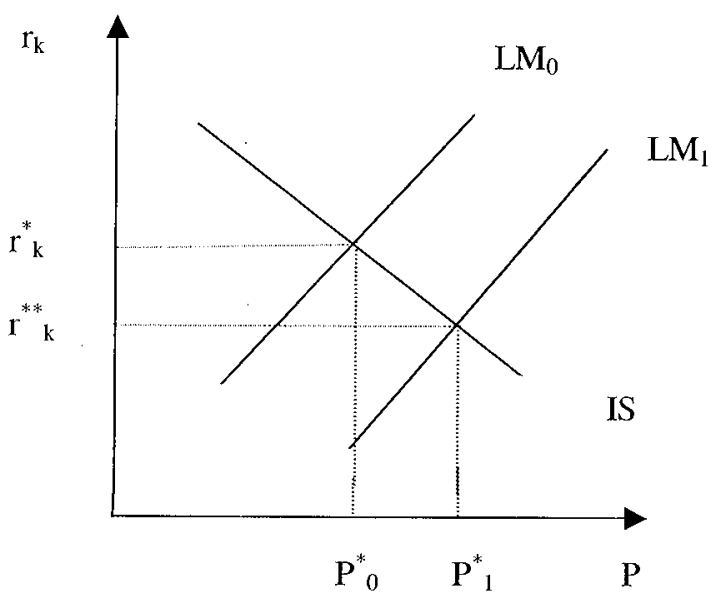

Deve-se ressaltar, mais uma vez, que estamos supondo que a economia opera com pleno emprego da força de trabalho, ou seja, que $Y$ $=Y_{f}$. A política monetária não pode, portanto, afetar o nível de emprego e de produto de equilibrio de longo prazo, mas pode afetar a taxa real de retorno dos ativos de capital e a composição do produto real. Isso ocorre porque uma expansão monetária leva os agentes econômicos a aumentar a compra de máquinas e equipamentos, ou seja, produz um aumento do investimento. Esse aumento no investimento desejado irá resultar numa redução da taxa real de retorno do capital. Daqui se segue que a expansão monetária deverá produzir um aumento da taxa de acumulação de capital e, dessa forma, um aumento do crescimento de longo prazo.

A expansão monetária também resultou em preços mais altos, ou seja, numa redução do poder de compra da moeda. Esse é o aspecto negativo da expansão monetária no longo prazo. Do ponto de vista da formulação de política econômica, deve-se contrapor o 
custo inflacionário da expansão monetária com o benefício da mesma em termos de aceleração do crescimento. Qual dos dois efeitos é mais forte é uma questão empírica, não podendo ser respondida de forma apriorística.

\section{Conclusão}

Ao longo do presente artigo, argumentamos que a tese neoclássica de que a moeda é não-neutra apenas no curto prazo se apóia na hipótese de que a produtividade marginal do capital - ou, em modelos Keynesianos, a eficiência marginal do capital - é constante e independente da escolha de portfólio dos agentes econômicos. Contudo, se admitirmos que a eficiência marginal do capital é uma função decrescente do nível geral de preços, então uma expansão monetária irá produzir um aumento do nível de investimento e uma redução da taxa real de retorno dos ativos de capital, mesmo no caso em que a economia se encontra operando com plena utilização da capacidade produtiva. Daqui se segue que a política monetária pode influenciar a composição do produto real no longo prazo e, dessa forma, pode ser usada para induzir uma maior taxa de crescimento do produto. Esse aumento do crescimento, contudo, vem acompanhado por um aumento do nível geral de preços, ou seja, pela inflação.

Nesse contexto, a desejabilidade de uma política monetária expansiva deve ser avaliada em termos da comparação entre o custo inflacionário da mesma e o benefício proporcionado por uma maior taxa de crescimento. Essa avaliação não pode, contudo, ser feita em bases estritamente teóricas, sendo um problema de natureza empírica.

\section{Referências Bibliográficas}

CARVALHO, F.C. Mr. Keynes and the Post Keynesians. Aldershot: Edward Elgar, 1992C.

. On Bank 's Liquidity Preference. Anais do VInternational Workshop in Post Keynesian Economics, Knoxville, 1998.

CHICK, V. Macroeconomics After Keynes. MIT Press: Cambridge (Mass.), 1983. 
DAVIDSON, P. Money, Portfolio Balance, Capital Accumulation and Economic Growth. Econometrica, 367, 2, 1968.

DAVIDSON, P. Money and the Real World. $2^{\circ}$ Edição. Londres: Macmillan, 1978.

1994. Post Keynesian Macroeconomic Theory. Aldershot: Edward Elgar,

DOW, S. Money and the Economic Process. Aldershot: Edward Elgar, 1993.

HAHN, F. Equilibrium and Macroeconomics. MIT Press: Cambridge (Mass.), 1982a. - Dinero e Inflacion. Antoni Bosch: Barcelona, 1982b.

. Liquidity in FRIEDMAN, B; HAHN, F. Handbook of Monetary Economics. Londres: Elsevier Science Publishers, 1990.

HARRIS, L. Teoria Monetária. México: Fondo de Cultura, 1981.

HICKS, J. R. The Crisis in Keynesian Economics. Nova Iorque: Basic Books, 1974. . Dinéro, Interés Y Salários. México: Fondo de Cultura, 1989.

HIRSHLEIFER, J. Liquidity, Uncertainty and the Accumulation of Information in CARTER, C.F; FORD, J.L. (eds.). Uncertaintyand Expectations in Economics. Oxford: Basil Blackwell, 1972.

KAHN, R. Some Notes in Liquidity Preference in: Selected Essays on Employment and Growth. Cambridge: Cambridge University Press, 1974.

KEYNES, J. M. The General Theory of Employment, Interest and Money. Londres: Macmillan, 1973 [ edição original: 1936].

The General Theory of Employment in the Collected Writings of John Maynard Keynes. Londres: Macmillan, 1973.

Teoria Geral do Emprego, do Juro e da Moeda. São Paulo: Atlas, 1982 [edição original: 1936].

The Collected Writings of John Maynard Keynes. Londres: Macmillan [no texto os volumes são referidos por CWJMK].

LIMA, G.T. Em Busca do Tempo Perdido: a recuperação Pós-Keynesiana da economia do emprego de Keynes. $16^{\circ}$ Prêmio BNDES de Economia, Rio de Janeiro, 1992.

McCALLUN, B. Monetary Economics. Nova Iorque: Macmillan, 1989.

Press, 1996.

. International Monetary Economics. Nova Iorque: Oxford University

MILGATE, M. Capital and Employment. Nova Iorque: Academic Press, 1982.

MODIGLIANI, F. Liquidity Preference and the Theory of Interest and Money. Econometrica, 12, 1944. 
ONO, Y. Money, Interest and Stagnation. Oxford: Clarendon Press, 1994.

PETTENATI, P. Alternative Theories of a Money-Capital Economy: Keynes, Tobin and Neoclassics. Oxford University Press, Vol. 29, 1977.

ROBINSON, J. A Model of Acumulation in SEN, A (org.). Growth Economics. Penguim Books: Middlesex, 1970 [ano da publicação do artigo: 1962].

ROGERS, C. Money, Interest and Capital. Cambridge University Press: Cambridge, 1989.

ROMER, D. (1996). Advanced Macroeconomics. McGraw Hill: Nova Iorque.

SARGENT, T. Macroeconomic Theory. Academic Press: San Diego, 1987.

Bounded Rationality in Macroeconomics. Oxford: Clarendon Press,

1993.

SAVAGE, L.J. The Foundations of Statistics. Nova Iorque: John Wiley, 1954.

SHACKLE, G.L.S. Expectation in Economics. Cambridge: Cambridge University Press, 1952.

University Press, 1961.

Decision, Order and Time in Human Affairs. Cambridge: Cambridge La Naturaleza del Pensamiento Econômico. Cidade do México: Fondo de Cultura, 1966.

Expectation, Enterprise and Profit. George Allen and Unwin, 1970.

SIDRAUSKI, M. Rational Choice and Patterns of Growth in a Monetary Economy. Journal of Political Economy, Vol. 75, $1967 \mathrm{a}$.

. Inflation and Economic Growth. American Economic Review, Vol. 57, No. 2, 1967b.

SOLOW, R. Contribution to the Theory of Economic Growth. The Quarterly Journal of Economics, Vol. 70, Fev. 1956.

TAKAYAMA, A. Analytical Methods in Economics. The University of Michigan Press: Michigan, 1993.

TOBIN, J. A Dynamic Aggregative Model in Essays in Economics, Vol. 1, Macroeconomics. Cambridge: MIT Press, 1987 [ano da publicação do artigo: 1955].

. Liquidity Preference as a Behavior Toward Risk in Essays in Economics,

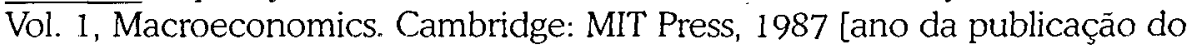
artigo: 1958].

A General Equilibrium Approach to Monetary Theory in Essays in Economics, Vol. 1, Macroeconomics. Cambridge: MIT Press, 1987 [ano da publicação do artigo: 1969]. 
. Asset Accumulation and Economic Activity. Chicago: the University of Chicago Press, 1981. . Money, Credit and Capita. Nova Iorque: McGraw Hill, 1998.

TOBIN, J; BRAINARD, C. Asset Markets and the Cost of Capital in TOBIN, J. Essays in Economics: theory and policy. Cambridge (mass.): MIT Press, 1982 [ano da publicação do artigo 1969].

VON NEUMANN; MORGENSTERN, O. Theory of Games and Economic Behavior. Princeton: Princeton University Press, 1947. 